\title{
ACComplex - Aplicativo para aprendizagem de Análilse de Circuitos em Corrente Alternada
}

\author{
Marcelo A. Corrêa \\ Departamento de Processos Industriais - Campus Maceió - Instituto Federal de Alagoas \\ $-($ IFAL) \\ R. Mizael Domingues, 75 - Centro, 57020-600- Maceió - AL - Brazil \\ marcelo@gmail.com
}

\begin{abstract}
An application to help learning mathematics with Complex Numbers and their application in circuit analysis of alternating current is the main objective of this work. The application is intended to be a simulation tool in the field of mathematics assisting learning in the conversion of Complex Numbers in Rectangular and Polar notation, as well as virtual load test and graphic representation of the complex power applied in the triangle of potency on circuit of alternating current.
\end{abstract}

Resumo. Um aplicativo para auxiliar a aprendizagem da Matemática com Números Complexos e sua aplicação em Análise de Circuitos Elétricos em Corrente Alternada é o objetivo principal deste trabalho. Por isto este aplicativo pretende ser uma ferramenta de simulação no campo da matemática auxiliando a aprendizagem na conversão de Números Complexos em notação Retangular e Polar, além de simular também a aplicação em circuitos de corrente alternada, com ensaio virtual de cargas, cálculo de corrente, potência complexa e representação gráfica para o triângulo de potência.

\section{Cenário de Uso}

O Instituto Federal de Alagoas (IFAL), instituição que oferta educação profissional técnica de nível médio tem o desafio de trabalhar com alunos provenientes do ensino fundamental com deficiências de aprendizagem em português e matemática.

Como confirmam os dados dos resultados da Prova Brasil 2013 (http://www.qedu.org.br/brasil/aprendizado), onde em matemática, 11\% é a proporção de alunos que aprenderam o adequado na competência de resolução de problemas e, em português, é de $23 \%$ a proporção de alunos que aprenderam o adequado na competência de leitura e interpretação de texto, até o $9^{\circ}$ ano na rede pública de ensino. Tal cenário dificulta desenvolver conceitos, habilidades e competências necessárias a formação do técnico em eletrotécnica e eletrônica.

O perfil dos alunos do curso técnico integrado de eletrotécnica e eletrônica, do IFAL, campus Maceió, é caracterizado por adolescentes na faixa etária de 14 a 18 anos que fazem uso intensivo de aparatos tecnológicos como games, tablets, smartphones e notebooks.

Um dos desafios enfrentados por estes alunos é a compreensão de Cálculos Matemáticos com Números Complexos utilizando notação retangular e polar, tópico 
imprescindível para a aprendizagem de conceitos de potência complexa e sua aplicação em análise de circuitos em corrente alternada.

Como defende Meyer e Rose2 (2014) em seu livro "Universal Design for Learning", a aprendizagem mediada pelas novas tecnologias da comunicação e informação ocorre na interação dinâmica entre os estudantes, o ambiente de aprendizagem e o contexto, de forma dinâmica e complexa. Para o autor, as ferramentas tecnológicas fazem parte do ambiente de aprendizagem, e, para tanto, tais ambientes devem ser concebidos de tal forma que promovam a interação e a colaboração entre os envolvidos no processo de aprendizagem.

Neste sentido é que o aplicativo Accomplex foi pensado e desenvolvido, como um objeto de aprendizagem da Matemática com Números Complexos e simulação de circuitos de corrente alternada, com ensaio virtual de cargas, cálculo de corrente, potência complexa e representação gráfica para o triângulo de potência.

Atualmente o aplicativo está com sua versão Beta aplicada aos Cursos Técnicos de Eletrotécnica e Eletrônica do IFAL-Campus Maceió, apresentando bons resultados na melhora do processo de aprendizagem dos alunos, servindo também de espaço de testes, validação e retroalimentação para o aperfeiçoamento das funcionalidades e interface gráfica do aplicativo.

\section{Desenvolvimento}

O Grupo de Pesquisa em Redes Inteligentes desenvolve trabalhos buscando a inovação em processos e produtos na área de Educação Técnica e Tecnológica. Este ambiante possibilitou a criação de um applicativo para uso em smartphones com Android, orientado para o ensino e a aprendizagem em Análise de Circuitos Elétricos focando o ensino técnico e tecnológico.

O aplicativo está sendo desenvolvido no ambiente SDK Android Studio 2.3.3, utilizando linguagem JAVA, a partir da modelagem em UML das principais funcionalidades do software. Para acelerar o processo de desenvolvimento de um protótipo, optou-se pela utilização do ambiente App Inventor que é uma plataforma web de código aberto, mantida pelo Massachusetts Institute of Technology (MIT), o que levou a um maior envolvimento dos alunos, que atualmente estão interessados em implementar novas funcionalidades ao aplicativo. A modelagem em UML foi desenvolvida na versão 1.4., com auxílio do ArgoUML, sendo que a validação das funções matemáticas desenvolvidas no aplicativo foram realizadas em duas etapas, a primeira partindo de um banco de resultados de problemas matemáticos propostos em livros e apostilas utilizadas no IFAL-Campus Maceió. Esta etapa validou a versão Beta que foi disponibilizada para os alunos.

\subsection{A Simulação como principal estratégia para aplicação de Apps no processo de aprendizagem.}

Uma das funcionalidades possíveis de implementação nos Apps é a simulação numérica, o que permite seu uso como ferramenta de auxílio à aprendizagem, uma vez que coloca a disposição do usuário a mobilidade para análise de resultados a qualquer tempo em qualquer lugar. Esta característica implica na utilização de modelos 
matemáticos abrindo um campo para aprendizagem na área de engenharia pela utilização de algoritmos com técnicas de cálculo numérico.

A modelagem matemática é uma área bem estruturada e consolidada na ciência, com um acervo técnico extenso e com várias modelos de simulação e técnicas que garantem convergência. Isto foi o que motivou a criação da funcionalidade de simulação de circuitos no App Accomplex, onde modelos consagrados das situações reais em que se encontram as cargas dispostas nas instalações residenciais, prediais ou industriais foi adotado.

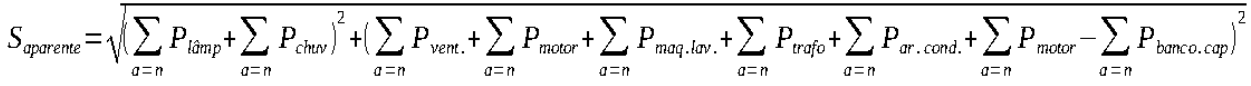

$$
\begin{aligned}
& \hat{A} \text { ngulo fi }=\arctan \left(\frac{\sum_{a=n} Q_{\text {reativo }}\left(P_{\text {vent. }}+P_{\text {maq. tav }}+P_{\text {trafo }}+P_{\text {ar. cond. }}+P_{\text {motor }}-P_{\text {banco. cap }}\right)}{\sum_{a=n} P_{\text {ativa }}\left(P_{\text {lamp }}+P_{\text {chuv. }}\right)}\right)
\end{aligned}
$$

\section{Figura 1. Modelagem matemática para o cálculo de Potencia Aparente e Ângulo de carga no simulador de cargas AC.}

O modelo matemático para análise de potência complexa foi a configuração de cargas em paralelo, o que possibilita duas configurações de entrada, uma considerando a alocação de equipamentos e outra considerando a inserção de parâmetros elétricos de impedância. Tal funcionalidade permite atingir a variabilidade na aprendizagem de análise de circuitos através da interação com os parâmetros de simulação, que permite o caminho por várias lógicas de raciocínio. Deste modo o interagente pode perceber a interdependência entre os parâmetros de entrada, por simulações repetidas em vários caminhos de raciocínio. Nesta perspectiva é que se defende a implementação de simuladores em aplicativos móveis, como ferramenta ao processo de aprendizagem no ensino médio.

\section{Apresentação do Software}

O Aplicativo Accomplex possui três seções distintas, dentro das quais encontram-se as funcionalidades de cálculo, simulação e representação gráfica para análise de potência elétrica em circuitos AC.

\section{- $1^{\text {a }}$ Seção: Conversão de Números Complexos}

Nesta seção é possível utilizar o aplicativo com uma calculadora científica com recursos gráficos. O usuário poderá converter números complexos entre as formas de representação retangular e polar, testando vários valores e observando graficamente e numericamente o resultado da conversão.

Os dois botões (Retang $\rightarrow$ Polar) e (Polar $\rightarrow$ Retang.) permitem acessar diretamente a tela de cada processo de conversão instantaneamente.

Esta seção foi orientada para auxiliar na aprendizagem do processo matemático envolvido com a habilidade em cálculos com números complexos.

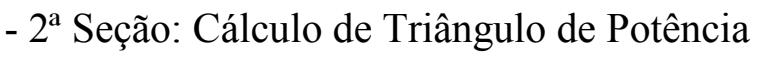


Nesta seção o aplicativo auxilia o aprendizado de Potências Elétricas em circuitos de corrente alternada, explorando o cálculo para análise de Potências Complexas, permitindo distingui-las nas suas três formas de configuração: Potência APARENTE, Potência ATIVA e Potência REATIVA.

O usuário poderá efetuar cálculos e simular o comportamento da variação de cada modo da potência através dos botôes (APARENTE)(ATIVA)(REATIVA).

$3^{\text {a }}$ Seção: Simulador de Circuitos AC

Esta seção foi desenvolvida com a simulação de situação reais de configuração de cargas elétricas em instalações residênciais, comerciais e industriais. A aprendizagem dos conceitos envolvidos em análise de circuitos em corrente alternada podem ser experimentadas em duas situações:

Análise de Cargas AC, selecionada pelo botão (Cargas AC) e Análise de Potência em circuitos modelados RLC paralelo, selecionada pelo botão (Análise de Potência).

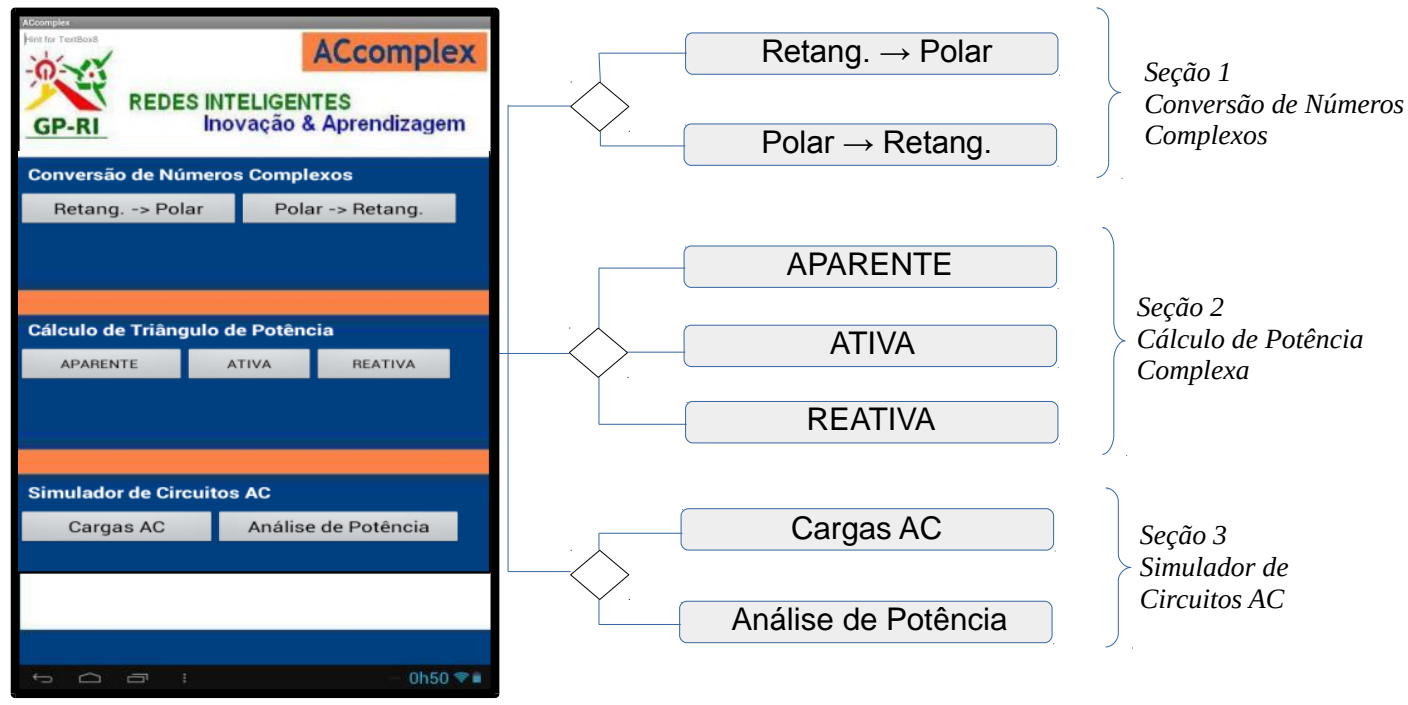

Figura 2. Tela de Abertura e Estrutura do Aplicativo Accomplex

\subsection{Seção 1 : Conversão de Números Complexox}

Esta função, acessada ao apertar o botão (Retang. $\rightarrow$ Polar) na função principal, permite converter um número complexo dado por "a" = $($ Real + Imaginário $x \mathrm{i})$ em um número complexo na forma Polar dado por Módulo e Ângulo. O gráfico com o círculo fará a projeção ortogonal e radial do número dado pelo usuário permitindo observar a relação entre as componentes Retangular e Polar.

A função, acessada ao apertar o botão (Polar $\rightarrow$ Retang.) na função principal, fara a função inversa do que foi apresentado acima, seguindo a mesma lógica de funcionalidade, fazendo a conversão de um número complexo dado por |Módulo| e Ângulo para a forma Retangular apresentando também o gráfico correspondente a conversão. 
CBIE-LACLO 2015

Anais dos Workshops do IV Congresso Brasileiro de Informática na Educação (CBIE 2015)

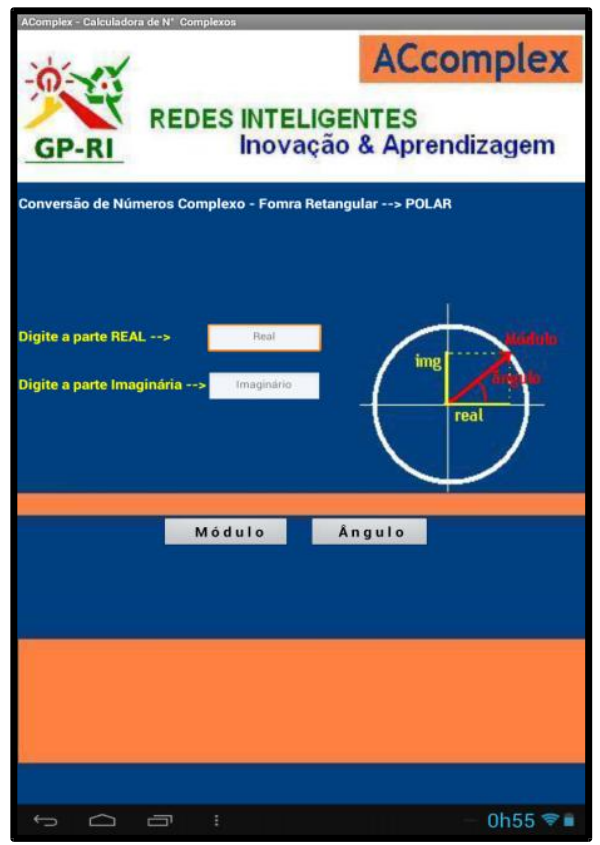

Figura 4. Tela da Funcionalidade de Conversão de Números Complexos Notação (Retang. $>$ Polar)

\subsection{Seção 2 : Cálculo de Triângulo de Potência}

A funcionalidade para Cálculos de Triângulo de Potência é ativada ao se pressionar um dos três botões (APARENTE)(ATIVA)(REATIVA).

Para cada tipo de potência é possível simular cálculos para configuração do triângulo de potência, sendo que a defasagem entre corrente e tensão é calculada através do ângulo entre a Potência Aparente e a Potência Ativa, e é expresso na forma de Cos fi, que representa também o Fator de Potência para a configuração apresentada.

Na função Cálculo de Triângulo de Potência $\rightarrow$ APARENTE, o aplicativo apresenta a solicitação de dados de Potência Ativa e Reativa, e através de relações trigonométricas realiza o cálculo da Potência Aparente e do fator de potência, expressando o resultado nas etiquetas amarelas. Para permitir uma maior compreensão sobre as interações entre as Potências o aplicativo traz a representação gráfica do triângulo de potência, proporcionalmente aos dados fornecidos, possibilitando que o usuário pratique várias simulações de dados e observe as implicações sobre o fator de potência, contribuindo na aprendizagem da análise de circuitos em corrente alternada.

Analogamente a função do Cálculo de Triângulo de Potência $\rightarrow$ APARENTE, as funções para Potência ATIVA e REATIVA assim operam, o que possibilita que o usuário transite entre as três configurações possíveis simulando situações reais de cargas em instalações elétricas.

Assim, as Seções 1 e 2 se complementam permitindo observar a relação entre a geometria da Notação Retangular e Polar dos Número Complexos com a geometria do Triângulo de Potência com intuito de conduzir o interagente para a Terceira Seção que contém o Simulador de Carga e Ciruitos. 
CBIE-LACLO 2015

Anais dos Workshops do IV Congresso Brasileiro de Informática na Educação (CBIE 2015)

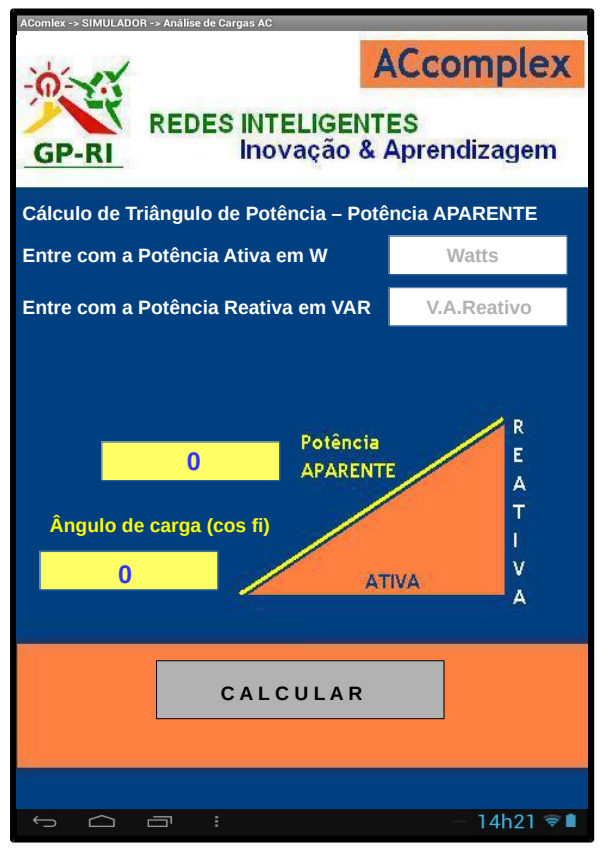

Figura 4. Tela da Funcionalidade Triângulo de Potência $>$ Aparente

\subsection{Seção 3: Simulador de Circuitos AC}

Nesta seção são possíveis dois tipos de simulações para análise de circuitos AC, uma atribui cargas em paralelos do tipo resistivas, indutivas e capacitivas de forma visual, e outra através do fornecimento de parâmetros elétricos como resistência, indutância e capacitância. Ao pressionar o botão (Cargas AC) na tela principal, será ativada a funcionalidade de simulação de cargas comuns em circuitos elétricos residenciais, prediais e comerciais conectadas em paralelo à fonte de alimentação AC.

Ao selecionar o ícone da carga que se quer adicionar, os quais estão disponíveis na barra principal como (lâmpadas, chuveiro, ventilador, máquina de lavar, transformadores, aperelhos de ar-condicionado, motores e banco de capacitores), o painel abaixo da barra irá apresentar a quantidade de cada tipo de carga adicionado. Levando em consideração o valor padrão de potência de cada item, são calculadas as seguintes relações de potência, corrente e ângulo de defasagem:

- Potência ATIVA em Watts: representando a parcela de potencia complexa (parte real) caracterizada pela adição de cargas resistivas.

- Potência REATIVA em VAR: representando a parcela de potência complexa (parte imaginária) caracterizada pela adição de cargas indutivas tomadas positivamente e cargas capacitivas negativamente.

- Potência APARENTE em VA: representando a parcela de potência complexa (módulo na forma polar) caracterizado pelo produto da tensão pela corrente no circuito.

Com os dados de potência o aplicativo utiliza o modelo do triângulo de potência para calcular a defasagem entre a corrente e tensão aplicada ao circuito fornecendo o ângulo em graus. 


\section{CBIE-LACLO 2015}

Anais dos Workshops do IV Congresso Brasileiro de Informática na Educação (CBIE 2015)

Com estes parâmetros calculados o aplicativo apresenta os resultados da simulação expressando o fator de potência para o circuito calculado a partir da função Cos(ângulo de defasagem), assumindo as duas possíbilidade de tensão de alimentação mais frequêntes que são $110 \mathrm{~V}$ e $220 \mathrm{~V}$.

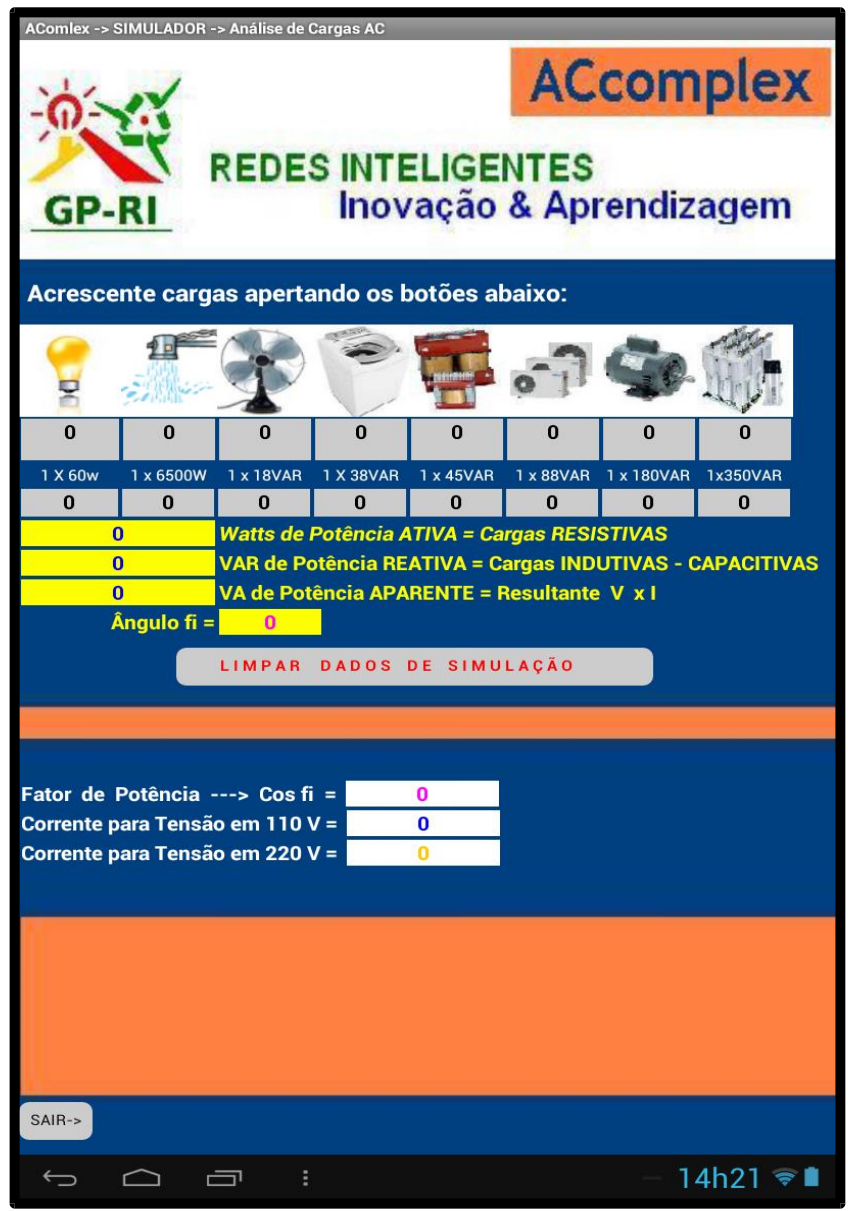

Figura 5. Tela do Simulador de Cargas AC

Esta funcionalidade permite que o usuário simule várias situações de carga e com a devida observação dos resultados compreenda conceitos de correção de fator de potência, bem como os principais parâmetros envolvidos na análise de circuitos em corrente alternada.

\section{Considerações finais}

Atualmente o aplicativo Accomplex está sendo utilizado por alunos dos cursos técnicos de Eletrotécnica e Eletrônica do IFAL Campus Maceió, onde disponibilizou-se uma versão Alfa.

A segunda etapa de validação do aplicativo, que foi planejada através da utilização do App na resolução de problemas matemáticos, gerou um verdadeiro ambiente de aprendizagem ao redor da estruturação de novas funcionalidades, o que culminou em um curso de programação no ambiente MIT AppInventor para os alunos que demonstraram interesse em fazer parte do desenvolvimento. 


\section{CBIE-LACLO 2015}

Anais dos Workshops do IV Congresso Brasileiro de Informática na Educação (CBIE 2015)

As funcionalidades propostas estão em estudo para serem implementadas, como a simulação para cálculo de impedância complexa e a adição de mais um módulo orientado para Projetos Elétricos, que permitiria o uso em outras disciplinas e instituições.

O foco atual é a melhoria da interface com a implementação de funções gráficas, que permitam o interagente variar parâmetros de circuitos e notações retangular e polar atuando diretamente sobre a variação de vetores, fazendo o aplicativo interpretar graficamente a entrada de dados.

As contribuições pedagógica foram perceptíveis nestes dois últimos semestres (2014-2 e 2015-1) onde o uso efetivo por parte dos alunos, medidos em numero de donwloads do App, foi constatado. Houve o aumento da participação dos alunos nas discussões realizadas em saula de aula, bem como o número de sugestões e defeitos que foram corrigidos ainda na versão Alfa, o que denota um avanço na aprendizagem, uma vez que as simulações servem de exercícios de fixação sobre o tema trabalhado em sala de aula.

Os alunos aderiram ao movimento de criação e expansão do aplicativo o que demonstra a verdadeira apropriação da tecnologia de uma forma realmente nova.

A expectativa agora é colher mais dados para lançamento da versão Beta, pois a meta para o próximo ano é desenvolver uma versão utilizando o ambiente Android Studio, programando-se todas as funcionalidades em Java. Pretende-se também ampliar a divulgação e a colaboração no aprimoramento e manutenção do código, uma vez que será aberto para uso, licenciado em Creative Commons.

Por estes resultados e com esta vivência, acredita-se na viabilidade do uso de Apps em processos educacionais, e seu uso na promoção de um ambiente que fomente a aprendizagem de conteúdos específicos que compõem o currículo do ensino médio.

\section{References}

${ }^{1}$ Dirksen, John, (2011) “ Design for How People Learn”, Editora New Riders, US

${ }^{2}$ Meyer, A. \& Rose, D. H. (2014) "Universal Design for Learning:Theory and Practice, CAST Professional Publishing. EUA.

Monteiro, J. B. (2012) "Google Android: crie aplicações para celulares e tablets", Editora Casa do Código, SP - Brasil.

Wei-Meng, L. (2011), "Introdução ao Desenvolvimento de Aplicativos Para o Android" (traduçaõ para português), Editora Ciência Moderna, RJ - Brasil.

Deitel, P. (2012) “Android for Programmers: An App-Driven Approach", (em português), Editora Bookman - Grupo A; SP-Brasil.

Dresch, A. (2014) "Design science research: método de pesquisa para avanço da ciência e tecnologia". Editora Bookman, Porto Alegre-RS-Brasil.

Mota, R. (2014) "Educando para inovação e aprendizagem independente; Editora Campus - Elsevier, RJ - Brasil. 